\title{
Extended Length of Hospital Stay for Surgical and Medical Patients - Insights from Hospital and Psychosocial Predictors
}

\author{
Chinedu I. Ossai \\ Swinburne University, Australia \\ cossai@swin.edu.au
}

\author{
Nilmini Wickramasinghe \\ Swinburne University Australia \& \\ Epworth Healthcare Australia. \\ nilmini.work@gmail.com
}

\begin{abstract}
Ensuring that patients do not overstay the expected Length of Stay (LOS) in the hospital is an important indicator of the quality of care and helps to reduce the cost of healthcare. This study identifies the predictors of Extended Length of Hospital Stay (ELOHS) for surgical and medical patients to include LOS (>20 days), Age (>40 years), Hour to Surgery (HTS) - within 4 hours of admission, zero and one Rapid Response Team (RRT) calls, Average Operating Room Time (AORT) of $0-120$ minutes and one Theatre Session (TS). Apart from the "ear, nose, mouth \& throat", "kidney and urinary tract", "circulatory system", "nervous system" and "digestive system" Major Diagnostic Categories (MDCs), other considered MDCs have significant differences in the Classification of Hospital Acquired Diagnoses (CHADx) rate for ELOHS and Normal Length of Hospital Stay (NLOHS) patients. It is expected that the early consideration of ELOHS predictors will be vital in improving patients' outcomes in the hospital
\end{abstract}

\section{Introduction}

Hospital admission is very important for saving lives through enhanced care for the sick with facilities that will ordinarily not be available in health centres. However, when patients overstay in the hospital, it starts to constitute problems for them, the hospital, and the wider economy due to the mounting costs [1-4]. Patients can develop other comorbidities and complications due to the numerous nosocomial infections [5] that have been shown to hamper the quality-of-life during and after hospital admissions [6].

Extended Length of Hospital Stay (ELOHS), which is described as patients overstaying the high trim point (3*average LOS) for a particular Diagnosis Related Group (DRG), has also contributed negatively to the hospital image because it has been used as one of the quality indicators of good hospital care [7]. Imperatively, affecting hospitals' insurance reimbursement because of the penalties imposed on them for poor patient care [8]. Numerous studies have looked at ELOHS for different disease conditions to identify the factors responsible for patients overstaying the time they are expected to stay in hospital. Passias et al. [9] studied the influencing factors of ELOHS for cervical spondylotic myelopathy and showed that age, diabetes, posterior surgical approach, and operative time are among the contributors. ELOHS amongst patients that underwent hindfoot arthrodesis procedure were identified to include diabetes neuropathy, external fixation and infections, and external factors such as possession of health insurance policy [10]. Cheng et al. [29] attributed ELOHS to older age, cognitive impairment, higher number of medical conditions requiring medication, and violence during hospital stay for psychiatric patients whereas Dial et al. [30] identified those older than 65 years, marital status, private health insurance, African American race, removal of iliac crest autograph used for spinal fusion as some of the psychosocial factors contributing to ELOHS. Marfil-Garza et al. [23] showed that younger age, male gender, a lower physician-to-patient ratio, emergency and weekend admissions, surgery, the number of comorbidities, residence outside the city and lower socioeconomic status are influencers of ELOHS.

ELOHS has been linked to increased hospitalization and decreased survival rate after carotid endarterectomy while being influenced by risk factors such as history of congestive heart failure, chronic obstructive pulmonary disease, total time in operating room, number of hospital visits, Intensive Care Unit (ICU) transfers, and placement of Foley catheter over [11]. Patients with deep sternal wound infection have been identified to overstay in hospitals due to factors that include diabetes, obesity, heart failure, renal impairment, and complex surgical procedures [12]. Patients with unruptured adult cerebral aneurysms have risk factors of ELOHS that comprise demographics factors, preadmission comorbidities, choice of procedures, and inpatient complications [3]. For the elderly patients in the acute care hospital context, ELOHS is influenced by tube feeding, consumption of five or more medications, non-independent status, urinary tract infection, pneumonia, congestive heart failure, and hypoalbuminemia [13].

Despite the myriads of studies and the findings, ELOHS has not been considered holistically to allow hospitals to tailor down their focus on patients of specific characteristics to improve care and facilitate recovery. Even though many studies have relied on the ELOHS that 
varied from 1 - 9 days for different DRGs [9, 14-16], they have produced DRG specific risk factors that failed to consider a wide range of hospital-specific predictors. Looking at the fact that complication rates in hospitals are always higher for patients that overstayed than those with a normal length of stay [31], it becomes necessary to understand how the influencers of ELOHS impact Hospital-acquired complications (HACs). Since the use of Classification of Hospital Acquired Diagnoses (CHADx) to measure HAC for patients [32] makes it easier to narrow the hospital complications by identifying, counting, and observing the numerous adverse events on patients during admission to improve their safety [33], it suffices to understand how ELOHS, and the factors studied in this study can influence CHADx. Because the importance of having a bigger picture of patients' status in managing ELOHS cannot be overemphasized and knowing the hospital-specific factors can help caregiving ab initio, this study intends to answer the following research questions.

- What are the psychosocial and hospital-specific predictors of ELOHS for surgical and medical patients undergoing various treatments in the hospital?

- What is the relationship between these risk factors?

- How does ELOHS impact the CHADx for patients of different age groups?

To answer these questions involves analyzing available medical records, categorizing the hospital and psychosocial traits of the patients, and ensuring that only hospital-based factors relating to patient's admission are considered. This will help to give administrators an indication of the outlier conditions that need better control in patients' management to forestall their contributions to ELOHS.

This study, therefore, aims to use the medical records of patients from an academic hospital to determine the hospital and psychosocial predictors of ELOHS for surgical and medical patients. The major contribution of this study is separately identifying the risk factors of ELOHS for surgical and medical patients in a hospital context where patients of numerous DRGs are treated at the same time. By relying on hospital-specific factors, which include VMO specialty, admission category, distance to hospital, socioeconomic status, etc., this study can bring a new dimension to the understanding of ELOHS predictors, hence giving room for prioritizing patients' management on admission. This will help to minimize overstaying in hospitals for the patients that are most vulnerable to ELOHS.

\section{Methods}

This study relied on de-identified data from an academic hospital situated in Melbourne Australia for the retrospective analysis of ELOHS for surgical and medical patients admitted between 10/2015 - 12/2020. Surgical patients were identified as those who had time in the operating theatre for any procedure whereas medical patients were not sent to the operating theatre. The patients with ELOHS are those identified as staying more than the high trim point ( $3 *$ average LOS) for a Diagnosis Related Group (DRG) whereas those with Normal Length of Hospital Stay (NLOHS) did not overstay the high trim point of their DRG despite the LOS in hospital.

The features used for the analysis include Visiting Medical Office (VMO) Specialty, Patient Age, Patient Gender, Admission Category (ADC), Patient LOS, Hours to Surgery (HTS), MDC Details, Patient Care Class, Average Operating Room Time (AORT) in minutes, Charlson Score (CS), Unplanned readmission (UPR), CHADx, Hospital-Acquired Complications (HACs), Return to Operating Room (ROR), ICU transfer, Transfusion (TRAF), Theatre Sessions (TS), Rapid Respond Team (RRT) calls, Day of Admission Surgery Patients (DOP) and Postcode. The postcode was used in conjunction with the socio-economic indexes for areas (SEIFA) from the Australian Bureau of Statistics (ABS) [17] to determine the Socioeconomic Status (SES) of the patients by ranking them as low (1-4 decile), middle (5-7 decile) and high (8-10 decile). The patient's distance to the hospital (DTH) was determined by using the Global Positioning System (GPS) distance calculation that relies on the longitudes, and latitudes of the patient postcode and the hospital postcode.

\subsection{Statistical Analysis}

The study relies on descriptive statistics, Pearson Chisquared and correlation coefficient analysis, and multivariate regression analysis for establishing the frequencies of occurrence and interrelationship between the features for ELOHS and NLOHS of patients. The Odds Ratio (OR), which depicts the likelihood of ELOHS is determined by using the dichotomous yes (ELOHS) or no (NLOHS) classification of patients' episodes from the retrospective records using the high trim points of the DRGs as the boundaries. Thus, patients who overstayed the high trim points are classified as yes whereas those who did not overstay are classified as no. To establish the statistical significance of the rate of hospital complications on admission, the Pearson Chi-squared analysis was used to determine the statistical significance of the CHADx between the ELOHS and NLOHS patients. The rate of patients' CHADx for various age groups namely, $<18$ years, $18-40$ years, $40-65$ years, and $\geq 65$ years was used for establishing the statistical significance difference at a 95\% confidence interval. The correlation coefficient is used to establish the influence the studied features have on each other to understand how the changes in each one for both ELOHS and NLOHS influence others.

\section{Results \\ 3.1 Descriptive Statistics of Predictors}

The analysis is done on 21926 surgical and 11826 medical patients records, which has $46 \%$ male and $54 \%$ 
female for the surgical patients, and $36 \%$ male and $64 \%$ female medical patients. The mean ages of the surgical patients are male $-64.53 \pm 20.46$ years and female - 61.57 \pm 21.71 years whereas medical patients have the mean ages of male and female as $69.36 \pm 23.86$ and $66.05 \pm 25.37$, respectively. The AORT and LOS of surgical patients are - AORT: male $-65.12 \pm 62.10 \mathrm{mins}$, female $-65.63 \pm 58.10$ mins; LOS male $-5.91 \pm 10.74$ days, female $-6.28 \pm 9.98$ days. The LOS of medical patients are - male $6.22 \pm 8.32$ days and female $6.99 \pm 8.72$ days while the ELOHS rate is $12.41 \%$ and $9.94 \%$, respectively for surgical and medical patients.

The various MDCs admitted between $0-1.79 \%$ and $0.14-1.36 \%$ of surgical and medical patients respectively, who overstayed their LOS in the hospital The VMO specialties admissions for patients with NLOHS are $0.26-13.17 \%( \pm 3.22 \%)$ for surgical patients and $0.69-13.17 \%( \pm 3.11 \%)$ for medical patients. The summary of some descriptive statistics of some parameters used for the analysis is shown in Table 1 whereas the high trim points for the MDCs and the various DRGs are shown in Table 2. Please note that some psychosocial and hospital parameters such as VMO specialties, SES of patients, and DTH are not included in Table 1 due to the need to optimize space and reduce redundancy.

Table 1: Descriptive statistics (count, \%) of some of the features used for predicting the ELOHS and NLOHS for surgical and medical patients

\begin{tabular}{|c|c|c|c|c|}
\hline \multirow[t]{2}{*}{ Parameters } & NLOHS & ELOHS & NLOHS & ELOHS \\
\hline & \multicolumn{2}{|c|}{ Surgical Patients } & \multicolumn{2}{|c|}{ Medical Patients } \\
\hline Population & \multicolumn{2}{|c|}{21926} & \multicolumn{2}{|c|}{11826} \\
\hline ELOHS rate & \multicolumn{2}{|c|}{$12.41 \%$} & \multicolumn{2}{|c|}{$9.94 \%$} \\
\hline \multicolumn{5}{|c|}{ Patient Age } \\
\hline under_18 & $933(98.11 \%)$ & $18(1.89 \%)$ & $548(96.31 \%)$ & $21(3.69 \%)$ \\
\hline $18-40$ & $2429(94.55 \%)$ & $140(5.45 \%)$ & $1698(97.31 \%)$ & $47(2.69 \%)$ \\
\hline $40-65$ & $5663(91.38 \%)$ & $534(8.62 \%)$ & $1691(93.68 \%)$ & $114(6.32 \%)$ \\
\hline 65 and over & $10180(83.38 \%)$ & $2029(16.62 \%)$ & $6713(87.1 \%)$ & $994(12.9 \%)$ \\
\hline \multicolumn{5}{|c|}{ Patient Gender } \\
\hline Female & $10179(86.48 \%)$ & $1591(13.52 \%)$ & $6733(89.2 \%)$ & $815(10.8 \%)$ \\
\hline Male & $9026(88.87 \%)$ & $1130(11.13 \%)$ & $3917(91.56 \%)$ & $361(8.44 \%)$ \\
\hline \multicolumn{5}{|c|}{ Patient Length of Stay (LOS) } \\
\hline$\leq 5$ days & $15600(99.43 \%)$ & $90(0.57 \%)$ & $7828(99.92 \%)$ & $6(0.08 \%)$ \\
\hline 6-10days & $2319(71.22 \%)$ & $937(28.78 \%)$ & $1969(94.53 \%)$ & $114(5.47 \%)$ \\
\hline 11-20days & $942(51.96 \%)$ & $871(48.04 \%)$ & $667(53.83 \%)$ & $572(46.17 \%)$ \\
\hline$>20$ days & $344(29.48 \%)$ & $823(70.52 \%)$ & $186(27.76 \%)$ & $484(72.24 \%)$ \\
\hline \multicolumn{5}{|c|}{ Major Diagnostic Category (MDC) } \\
\hline $\begin{array}{l}\text { BLOOD, BLOOD FORM ORGANS, } \\
\text { IMMUNOLOG }\end{array}$ & $158(84.49 \%)$ & $29(15.51 \%)$ & $240(90.91 \%)$ & $24(9.09 \%)$ \\
\hline CIRCULATORY SYSTEM & $2809(86.01 \%)$ & $457(13.99 \%)$ & $1343(89.95 \%)$ & $150(10.05 \%)$ \\
\hline DIGESTIVE SYSTEM & $2823(85.18 \%)$ & $491(14.82 \%)$ & $1308(90.02 \%)$ & $145(9.98 \%)$ \\
\hline EAR, NOSE, MOUTH \& THROAT & $891(93.89 \%)$ & $58(6.11 \%)$ & $551(86.09 \%)$ & $89(13.91 \%)$ \\
\hline ENDOCRINE, NUTRITIONAL \& & $624(91.9 \%)$ & $55(8.1 \%)$ & $192(89.3 \%)$ & $23(10.7 \%)$ \\
\hline \multicolumn{5}{|l|}{ METABOLIC } \\
\hline HEPATOBILIARY SYSTEM \& PANCREAS & $712(85.58 \%)$ & $120(14.42 \%)$ & $150(93.75 \%)$ & $10(6.25 \%)$ \\
\hline INFECTIOUS \& PARASITIC DISEASES & $140(74.07 \%)$ & $49(25.93 \%)$ & $430(89.03 \%)$ & $53(10.97 \%)$ \\
\hline INJURY, POISON \& TOXIC EFFECT DRUGS & $313(82.37 \%)$ & $67(17.63 \%)$ & $279(90 \%)$ & $31(10 \%)$ \\
\hline KIDNEY \& URINARY TRACT & $1303(86.64 \%)$ & $201(13.36 \%)$ & $637(89.47 \%)$ & $75(10.53 \%)$ \\
\hline MUSCULOSKELETAL SYS \& CONN & $3809(88.56 \%)$ & $492(11.44 \%)$ & $914(88.65 \%)$ & $117(11.35 \%)$ \\
\hline \multicolumn{5}{|l|}{ TISSUE } \\
\hline NEOPLASTIC DISORDERS & $108(70.13 \%)$ & $46(29.87 \%)$ & $160(88.89 \%)$ & $20(11.11 \%)$ \\
\hline NERVOUS SYSTEM & $539(83.96 \%)$ & $103(16.04 \%)$ & $928(86.97 \%)$ & $139(13.03 \%)$ \\
\hline PREGNANCY, CHILDBIRTH \& & $782(98.36 \%)$ & $13(1.64 \%)$ & $1161(99.23 \%)$ & $9(0.77 \%)$ \\
\hline \multicolumn{5}{|l|}{ PUERPERIUM } \\
\hline RESPIRATORY SYSTEM & $639(90.25 \%)$ & $69(9.75 \%)$ & $1386(94.41 \%)$ & $82(5.59 \%)$ \\
\hline SKIN, SUBCUTANEOUS TISSUE \& BREAST & $1422(84.14 \%)$ & $268(15.86 \%)$ & $423(91.96 \%)$ & $37(8.04 \%)$ \\
\hline \multicolumn{5}{|c|}{ Classification of Hospital-acquired Diagnosis (CHADx) } \\
\hline No & $16119(91.28 \%)$ & $1540(8.72 \%)$ & $8687(91.98 \%)$ & $757(8.02 \%)$ \\
\hline Yes & $3086(72.32 \%)$ & $1181(27.68 \%)$ & $1963(82.41 \%)$ & $419(17.59 \%)$ \\
\hline \multicolumn{5}{|c|}{ Charlson Score (CS) } \\
\hline $0-1$ & $6901(93.19 \%)$ & $504(6.81 \%)$ & $3323(95.87 \%)$ & $143(4.13 \%)$ \\
\hline $2-4$ & $9966(87.1 \%)$ & $1476(12.9 \%)$ & $5021(88.18 \%)$ & $673(11.82 \%)$ \\
\hline $5-8$ & $1992(75.66 \%)$ & $641(24.34 \%)$ & $1956(86.51 \%)$ & $305(13.49 \%)$ \\
\hline$>8$ & $346(77.58 \%)$ & $100(22.42 \%)$ & $350(86.42 \%)$ & $55(13.58 \%)$ \\
\hline \multicolumn{5}{|c|}{ Average Operating Theatre Time -AOTT (min) } \\
\hline $0-60$ & $11159(86.25 \%)$ & $1779(13.75 \%)$ & & \\
\hline $60-120$ & $5210(89.95 \%)$ & $582(10.05 \%)$ & & \\
\hline$>120$ & $2836(88.74 \%)$ & $360(11.26 \%)$ & & \\
\hline
\end{tabular}




\begin{tabular}{|c|c|c|c|c|}
\hline \multicolumn{5}{|c|}{ VMO (Visiting Medical Officer) Specialty } \\
\hline Cardiology & $2028(87.79 \%)$ & $282(12.21 \%)$ & $842(91.32 \%)$ & $80(8.68 \%)$ \\
\hline Colorectal Surgery & $1187(86.64 \%)$ & $183(13.36 \%)$ & $247(92.86 \%)$ & $19(7.14 \%)$ \\
\hline Endocrinology & $58(53.21 \%)$ & $51(46.79 \%)$ & $58(53.21 \%)$ & $51(46.79 \%)$ \\
\hline Gastroenterology & $980(85.07 \%)$ & $172(14.93 \%)$ & $980(85.07 \%)$ & $172(14.93 \%)$ \\
\hline Gynecology & $394(92.49 \%)$ & $32(7.51 \%)$ & $394(92.49 \%)$ & $32(7.51 \%)$ \\
\hline Hematology & $129(65.82 \%)$ & $67(34.18 \%)$ & $129(65.82 \%)$ & $67(34.18 \%)$ \\
\hline Medical Oncology & $251(69.53 \%)$ & $110(30.47 \%)$ & $251(69.53 \%)$ & $110(30.47 \%)$ \\
\hline Nephrology & $137(45.67 \%)$ & $163(54.33 \%)$ & $137(45.67 \%)$ & $163(54.33 \%)$ \\
\hline Neurology & $83(51.88 \%)$ & $77(48.13 \%)$ & $83(51.88 \%)$ & $77(48.13 \%)$ \\
\hline Neurosurgery & $1085(95.51 \%)$ & $51(4.49 \%)$ & $1085(95.51 \%)$ & $51(4.49 \%)$ \\
\hline Obstetrics \& Gynae & $938(98.01 \%)$ & $19(1.99 \%)$ & $938(98.01 \%)$ & $19(1.99 \%)$ \\
\hline Orthopedic Surgery & $2888(90.59 \%)$ & $300(9.41 \%)$ & $2888(90.59 \%)$ & $300(9.41 \%)$ \\
\hline \multicolumn{5}{|c|}{ Distant to Hospital (DTH) } \\
\hline$>20 \mathrm{~km}$ & $5232(89.53 \%)$ & $612(10.47 \%)$ & $1121(88.9 \%)$ & $140(11.1 \%)$ \\
\hline $5-10 \mathrm{~km}$ & $4686(87.36 \%)$ & $678(12.64 \%)$ & $3002(89.96 \%)$ & $335(10.04 \%)$ \\
\hline $0-5 \mathrm{~km}$ & $5440(85.97 \%)$ & $888(14.03 \%)$ & $4467(91 \%)$ & $442(9 \%)$ \\
\hline $10-20 \mathrm{~km}$ & $3847(87.63 \%)$ & $543(12.37 \%)$ & $2060(88.83 \%)$ & $259(11.17 \%)$ \\
\hline \multicolumn{5}{|c|}{ Socioeconomic status (SES) } \\
\hline High & $15230(87.31 \%)$ & $2213(12.69 \%)$ & $9893(90.35 \%)$ & $1057(9.65 \%)$ \\
\hline Low & $1802(87.73 \%)$ & $252(12.27 \%)$ & $313(86.7 \%)$ & $48(13.3 \%)$ \\
\hline Middle & $2163(89.49 \%)$ & $254(10.51 \%)$ & $444(86.21 \%)$ & $71(13.79 \%)$ \\
\hline
\end{tabular}

Table 2: The Diagnosis Related Groups (DRGs) high trim point - $3 *$ average LOS $\left(Q_{3}\right)$ of the Length of Stay (LOS) of the various Major Diagnostic Categories (MDCs) and examples of DRG considered in the study.

\begin{tabular}{|c|c|c|c|}
\hline \multirow[t]{2}{*}{ MDC } & \multirow[t]{2}{*}{ DRG description } & \multicolumn{2}{|c|}{ DRG $\left(Q_{3}\right)$ in (days) } \\
\hline & & Mean \pm std & Min - Max \\
\hline Nervous System & $\begin{array}{l}\text { cranial and peripheral nerve disorders; degenerative nervous system } \\
\text { disorders; delirium; dementia and other chronic disturbances of } \\
\text { cerebral function; headache; nervous system neoplasm; nontraumatic } \\
\text { stupor and coma; seizure. stroke and other cerebrovascular disorders }\end{array}$ & $20.25 \pm 12.03$ & $6-58$ \\
\hline $\begin{array}{l}\text { Endocrine, Nutritional \& } \\
\text { Metabolic }\end{array}$ & $\begin{array}{l}\text { metabolic disorders; endocrine disorders; diabetes; severe nutritional } \\
\text { disturbance. }\end{array}$ & $19.26 \pm 9.41$ & $5-60$ \\
\hline Kidney \& Urinary Tract & $\begin{array}{l}\text { renal failure; kidney and urinary tract signs; urinary stones and } \\
\text { obstruction. }\end{array}$ & $15.93 \pm 10.48$ & $5-56$ \\
\hline $\begin{array}{l}\text { Male Reproductive } \\
\text { System }\end{array}$ & $\begin{array}{l}\text { inflammation and malignity of the male reproductive system; benign } \\
\text { prostatic hypertrophy. }\end{array}$ & $14.2 \pm 8.02$ & $4-31$ \\
\hline $\begin{array}{l}\text { Female Reproductive } \\
\text { System }\end{array}$ & $\begin{array}{l}\text { menstrual and other female reproductive system disorders; malignancy, } \\
\text { female reproductive system; infections, }\end{array}$ & $12.15 \pm 6.29$ & $4-33$ \\
\hline $\begin{array}{l}\text { Pregnancy, Childbirth \& } \\
\text { Puerperium }\end{array}$ & $\begin{array}{l}\text { vaginal delivery; antenatal and other obstetric admission; vaginal } \\
\text { delivery single uncomplicated; postpartum and post abortion. }\end{array}$ & $11.81 \pm 1.6$ & $3-18$ \\
\hline $\begin{array}{l}\text { Newborns \& Other } \\
\text { Neonates }\end{array}$ & neonate, admission wt. $2000-2499 \mathrm{~g}$ & $20.89 \pm 14.18$ & $7-60$ \\
\hline $\begin{array}{l}\text { Blood, Blood Form } \\
\text { Organs, Immunology }\end{array}$ & $\begin{array}{l}\text { reticuloendothelial and immunity disorders; red blood cell disorders; } \\
\text { coagulation disorders }\end{array}$ & $12.98 \pm 6.75$ & $7-31$ \\
\hline Neoplastic Disorders & lymphoma and non-acute leukemia; neoplastic disorders. & $16.64 \pm 11.5$ & $13-60$ \\
\hline $\begin{array}{l}\text { Infectious \& Parasitic } \\
\text { Diseases }\end{array}$ & $\begin{array}{l}\text { viral illness; septicemia; postoperative and post-traumatic infections; } \\
\text { fever of unknown origin; infectious and parasitic diseases. }\end{array}$ & $19.39 \pm 12.22$ & $9-60$ \\
\hline $\begin{array}{l}\text { Mental Diseases \& } \\
\text { Disorders }\end{array}$ & $\begin{array}{l}\text { anxiety disorders; personality disorders and acute reactions; major } \\
\text { affective disorders age }>69 \text {; }\end{array}$ & $29.65 \pm 18.88$ & $16-60$ \\
\hline $\begin{array}{l}\text { Eye Diseases \& } \\
\text { Disorders }\end{array}$ & $\begin{array}{l}\text { retinal procedures; hyphemia and medically managed trauma to the } \\
\text { eye; neurological and vascular disorders of the eye; acute and major } \\
\text { eye infections. }\end{array}$ & $13.95 \pm 6.12$ & $4-32$ \\
\hline $\begin{array}{l}\text { Alcohol/Drug Use } \\
\text { Disorders }\end{array}$ & drug intoxication and withdrawal & $31.11 \pm 14.55$ & $18-56$ \\
\hline $\begin{array}{l}\text { Injury, Poison \& Toxic } \\
\text { Effect Drugs }\end{array}$ & poisoning and toxic effect diagnosis; allergic reactions; injuries. & $15.49 \pm 8.46$ & $7-46$ \\
\hline $\begin{array}{l}\text { Factors Influencing } \\
\text { Health Status }\end{array}$ & surgical follow-up and medical care. & $14.63 \pm 6.06$ & $6-41$ \\
\hline $\begin{array}{l}\text { Ear, Nose, Mouth \& } \\
\text { Throat }\end{array}$ & $\begin{array}{l}\text { tonsillectomy and/or adenoidectomy; otitis media and URI; nasal } \\
\text { trauma and deformity; epistaxis; disequilibrium. }\end{array}$ & $9.49 \pm 2.89$ & $3-13$ \\
\hline Respiratory System & $\begin{array}{l}\text { whooping cough and acute bronchiolitis; respiratory system diagnosis; } \\
\text { respiratory neoplasms; respiratory infections/inflammations; pulmonary } \\
\text { embolism; chronic obstructive airways disease; bronchoscopy; }\end{array}$ & $21.66 \pm 9.13$ & $4-47$ \\
\hline
\end{tabular}




\begin{tabular}{|c|c|c|c|}
\hline & $\begin{array}{l}\text { pneumothorax; major chest trauma; interstitial lung disease; bronchitis } \\
\text { and asthma. }\end{array}$ & & \\
\hline Circulatory System & $\begin{array}{l}\text { venous thrombosis; valvular disorders; unstable angina; syncope and } \\
\text { collapse; peripheral vascular disorders; heart failure and shock; chest } \\
\text { pain; arrhythmia, cardiac arrest, and conduction disorders; } \\
\text { hypertension; heart failure and shock; arrhythmia, cardiac arrest, and } \\
\text { conduction disorders. }\end{array}$ & $15.56 \pm 10.34$ & $4-60$ \\
\hline Digestive System & $\begin{array}{l}\text { esophagitis and gastroenteritis; GI obstruction; digestive malignancy; } \\
\text { complex gastroscopy; abdominal pain or mesenteric adenitis; } \\
\text { inflammatory bowel disease; anal and stomal; abdominal pain or } \\
\text { mesenteric adenitis }\end{array}$ & $13.13 \pm 6.22$ & $4-46$ \\
\hline $\begin{array}{l}\text { Hepatobiliary System \& } \\
\text { Pancreas }\end{array}$ & $\begin{array}{l}\text { malignancy of hepatobiliary system, pancreas; hepatobiliary; disorders } \\
\text { of the biliary tract; }\end{array}$ & $16.93 \pm 8.62$ & $8-44$ \\
\hline $\begin{array}{l}\text { Musculoskeletal Sys \& } \\
\text { Conn Tissue }\end{array}$ & $\begin{array}{l}\text { sprains, strains, and dislocations of hip, pelvis, and thigh; non-surgical } \\
\text { spinal disorders; injury to the shoulder, arm, elbow, knee, leg, or ankle. } \\
\text { fractures of the neck of femur; distal femoral fractures; aftercare of } \\
\text { musculoskeletal implants; sprains, strains, and dislocations; spinal } \\
\text { fusion of hip, pelvis, and thigh; pathological fracture; musculoskeletal } \\
\text { malignant neoplasms; injury to forearm, wrist, hand, or foot. }\end{array}$ & $23.01 \pm 13.64$ & $4-60$ \\
\hline $\begin{array}{l}\text { Skin, Subcutaneous } \\
\text { Tissue \& Breast }\end{array}$ & $\begin{array}{l}\text { trauma to the skin, subcutaneous tissue, and breast; trauma to the skin, } \\
\text { subcutaneous tissue, and breast; malignant breast disorders; skin ulcers. }\end{array}$ & $19.7 \pm 9.96$ & $2-60$ \\
\hline
\end{tabular}

\subsection{Association of MDC with CHADx}

For the population of surgical patients admitted to the hospital, the CHADx rate of $27.68 \%$ is $10.09 \%$ higher than medical patients (with ELOHS rate of 17.59\%) admitted in the same period. The P-value of the Pearson Chi-squared between ELOHS and NLOHS for surgical and medical patients having CHADx is shown in Table 3. It can be deduced from Table 3 that most of the MDCs have the rate of CHADx amongst ELOHS and NLOHS not statistically significant at $P \leq 0.05$ because of the variation in patients' CHADx rates between them. Nonetheless, for medical patients suffering from "Nervous system" related MDC $(P$ $=0.0294$ ), and surgical patients suffering from "circulatory system" $(P=0.0018)$, "ear, nose, mouth \& throat" $(P=$ $0.0011)$, and "kidney \& urinary Tract" $(P<0.001)$ MDCs, there is no significant difference in the CHADx rates of the NLOHS and ELOHS cohorts. For surgical patients, apart from the "blood, blood from organs, immunology" and "factor influencing health status" MDCs that have 16 $20 \%$ more CHADx cases for ELOHS than NLOHS, other MDCs have more CHADx cases $(7-1100 \%)$ amongst NLOHS than ELOHS patients. The same is attenable with medical patients that have "factor influencing health status" and "ear, nose, mouth \& throat" MDCs that have 30 $-105 \%$ more ELOHS cases of CHADx than those NLOHS patients. Other MDCs have between $78.5-759 \%$ more cases of CHADx amongst NLOHS than ELOHS patients.

Table 3: Chi-square analysis for the rate of diagnosis in patients of various age groups with Classification of Hospital-acquired Diagnosis (CHADX) for various MDC amongst NLOHS and ELOHS (bold means significant at 95\% confidence level)

\begin{tabular}{llcl}
\hline MDC Details & NLOHS (count, \%) & ELOHS (count, \%) & P value \\
\hline Blood, Blood Form Organs, Immunology & Medical Patients & & 0.619 \\
Circulatory System & $25(0.21 \%)$ & $14(0.12 \%)$ & 0.987 \\
Digestive System & $184(1.56 \%)$ & $46(0.39 \%)$ & 0.09 \\
Ear, Nose, Mouth \& Throat & $118(1 \%)$ & $55(0.47 \%)$ & 0.125 \\
Endocrine, Nutritional \& Metabolic & $23(0.19 \%)$ & $30(0.25 \%)$ & 0.953 \\
Factors Influencing Health Status & $23(0.19 \%)$ & $9(0.08 \%)$ & 0.997 \\
Hepatobiliary System \& Pancreas & $18(0.15 \%)$ & $37(0.31 \%)$ & 0.812 \\
Infectious \& Parasitic Diseases & $16(0.14 \%)$ & $2(0.02 \%)$ & 0.217 \\
Injury, Poison \& Toxic Effect Drugs & $70(0.59 \%)$ & $22(0.19 \%)$ & 0.920 \\
Kidney \& Urinary Tract & $28(0.24 \%)$ & $10(0.08 \%)$ & 0.664 \\
Musculoskeletal Sys \& Conn Tissue & $82(0.69 \%)$ & $25(0.21 \%)$ & 0.911 \\
Neoplastic Disorders & $127(1.07 \%)$ & $47(0.4 \%)$ & 0.487 \\
Nervous System & $23(0.19 \%)$ & $10(0.08 \%)$ & $\mathbf{0 . 0 2 9}$ \\
Newborns \& Other Neonates & $110(0.93 \%)$ & $55(0.47 \%)$ & 0.899 \\
Pregnancy, Childbirth \& Puerperium & $36(0.3 \%)$ & $6(0.05 \%)$ & - \\
Respiratory System & $786(6.65 \%)$ & - & 0.397 \\
Skin, Subcutaneous Tissue \& Breast & $232(1.96 \%)$ & $27(0.23 \%)$ & 0.851 \\
\hline & $39(0.33 \%)$ & $12(0.1 \%)$ & \\
\hline Blood, Blood Form Organs, Immunology & Surgical Patients & & \\
Circulatory System & $12(0.05 \%)$ & $14(0.06 \%)$ & 0.9999 \\
Digestive System & $590(2.69 \%)$ & $174(0.79 \%)$ & 0.0838 \\
Ear, Nose, Mouth \& Throat & $559(2.55 \%)$ & $220(1 \%)$ &
\end{tabular}


Endocrine, Nutritional \& Metabolic

Eye Diseases \& Disorders

Factors Influencing Health Status

Female Reproductive System

Hepatobiliary System \& Pancreas

Infectious \& Parasitic Diseases

Injury, Poison \& Toxic Effect Drugs

Kidney \& Urinary Tract

Male Reproductive System

Musculoskeletal Sys \& Conn Tissue

Neoplastic Disorders

Nervous System

Pregnancy, Childbirth \& Puerperium

Respiratory System

Skin, Subcutaneous Tissue \& Breast

$\begin{array}{lll}69(0.31 \%) & 37(0.17 \%) & 0.4416 \\ 12(0.05 \%) & 1(0 \%) & 0.8557 \\ 10(0.05 \%) & 12(0.05 \%) & 0.6011 \\ 82(0.37 \%) & 26(0.12 \%) & 0.269 \\ 128(0.58 \%) & 63(0.29 \%) & 0.2638 \\ 45(0.21 \%) & 29(0.13 \%) & 0.3851 \\ 48(0.22 \%) & 34(0.16 \%) & 0.4959 \\ 134(0.61 \%) & 82(0.37 \%) & <\mathbf{0 . 0 0 1} \\ 87(0.4 \%) & 38(0.17 \%) & 0.2795 \\ 608(2.77 \%) & 210(0.96 \%) & 0.6606 \\ 32(0.15 \%) & 21(0.1 \%) & 0.6105 \\ 91(0.42 \%) & 53(0.24 \%) & 0.5148 \\ 252(1.15 \%) & 4(0.02 \%) & 0.0756 \\ 143(0.65 \%) & 29(0.13 \%) & 0.8609 \\ 91(0.42 \%) & 85(0.39 \%) & 0.8113\end{array}$

\subsection{Risk Factors of ELOHS}

Some of the risk factors of ELOHS for surgical and medical patients at a $95 \%$ significance level $(\mathrm{P} \leq$ 0.05) are shown in Table 4. Following Table 4, the predictors that have the likelihood of influencing ELOHS for surgical patients include LOS(>20days) - (OR:4.907, TS (once) - (OR:2.341, ROR (Yes) - (OR: 3.05), AORT (60 mins) - (OR:1.807), AORT (120 mins) - (OR: $1.622)$, Age $(\geq 65)$ - (OR:1.794), Age (40-65) (OR:1.501), HTS (4 hours) - (OR:1.621). Other parameters such as MDCs comprise "ear, nose, mouth \& throat" - (OR: 7.7499), "male reproductive system" (OR: 5.149), "circulatory system" - (OR: 1.995), "digestive system" - (OR:2.012), "factors influencing health status" - (OR: 2.894), etc., also influence ELOHS amongst surgical patients. For medical patients, the following parameters LOS (>20 days) - (OR:5.521), RRT (No calls) - (OR:4.69) and MDCs such as "ear, nose, mouth \& throat" - (OR: 7.0513), "factors influencing health status" - (OR: 2.632), "neoplastic disorders" - (OR: 3.952) and "digestive system" (OR:2.481) also have the probability of influencing ELOHS. Due to the smaller values of some of the other significant parameters $(\mathrm{OR}<1)$, they may have less likelihood of resulting in ELOHS amongst surgical and medical patients.

The accuracy of the multivariate Logistic model used to determine the risk factors of ELOHS is computed with the Receiver Operating Characteristics (ROC) Area Under the Curve (AUC) and confusion matrix computed for a $30 \%$ test data per Figure 1. The prediction accuracy of the surgical patients is $91.57 \%$ for training dataset and

Table 4: Significant Predictors of ELOHS with multivariate Logistic Regression analysis showing the Odds Ratio (OR), 95\% Confidence Interval (CI), and P-Value

\begin{tabular}{l|l|c}
\hline Features & Surgical Patients & Medical Patients \\
\hline ADC(Others) & OR:1.671,95\% CI $(1.143,2.442), \mathrm{P}=0.008$ & \\
ADC(PL1) & OR:0.775,95\% CI $(0.643,0.934), \mathrm{P}=0.007$ & - \\
ADC(UC1) & OR:0.771,95\% CI $(0.642,0.927), \mathrm{P}=0.006$ & - \\
ADC(MAT) & - & OR:0.072,95\% CI $(0.011,0.469), \mathrm{P}=0.006$ \\
AORT (60 mins) & OR: $1.807,95 \%$ CI $(1.494,2.185), \mathrm{P}<0.001$ & - \\
AORT (120 mins) & OR: $1.622,95 \%$ CI $(1.339,1.965), \mathrm{P}<0.001$ & - \\
CHADx (Yes) & OR:0.687,95\% CI $(0.594,0.795), \mathrm{P}<0.001$ & - \\
CS (>8) & OR:0.173,95\% CI $(0.118,0.255), \mathrm{P}<0.001$ & OR:0.184,95\% CI $(0.085,0.4), \mathrm{P}<0.001$ \\
CS $(2-4)$ & OR: $0.454,95 \%$ CI $(0.355,0.581), P<0.001$ & OR:0.444,95\% CI $(0.244,0.807), P=0.008$
\end{tabular}

92.43\% (AUC: 96\%) for the testing dataset whereas the accuracy for medical patients is $94.95 \%$ for the training dataset and $94.36 \%$ (AUC: $97 \%$ ) for the testing dataset. These accuracies show there is no overfitting because of the closeness of the training and testing prediction accuracies.

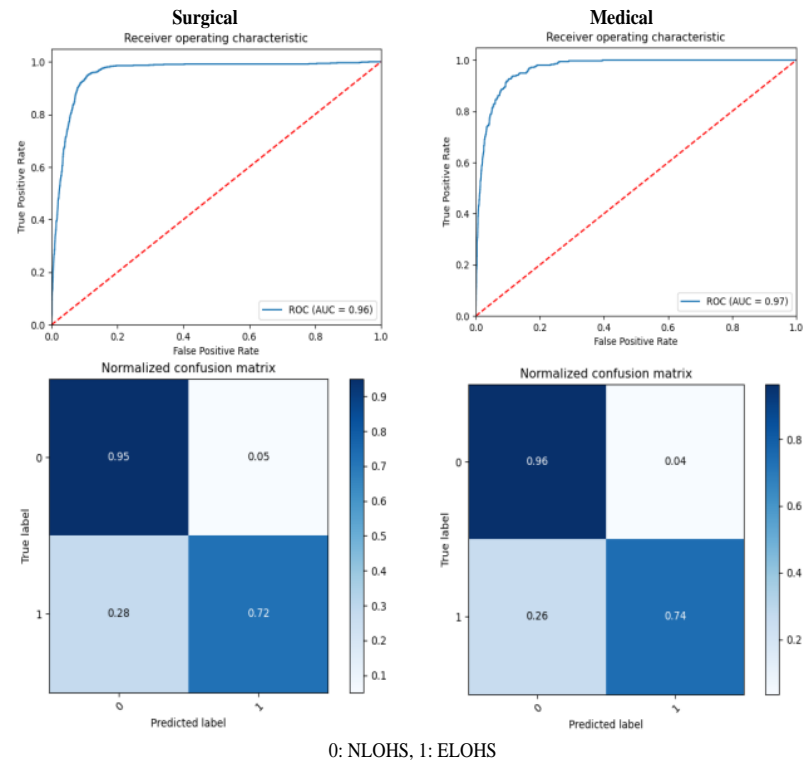

Figure 1: Summary of Logistic model prediction accuracy of $30 \%$ of the data used for testing 


CS (5-8)
DOP(Yes)
HACs (Yes)
HTS(4hrs)
ICU(Yes)
MDC
Circulatory System
Digestive System
Ear, Nose, Mouth \& Throat
Eye Diseases \& Disorders
Factors Influencing Health Status
Female Reproductive System
Hepatobiliary System \& Pancreas
Infectious \& Parasitic Diseases
Kidney \& Urinary Tract
Male Reproductive System
Skin, Subcutaneous Tissue \&
Breast
Neoplastic Disorders
Newborns \& Other Neonates
Respiratory System
Age (40-65)
Age ( $\geq 65$ )
LOS(>20days)
LOS( $\leq 5$ days)
LOS(6-10days)
ROR(Yes)
RRT (one call)
RRT(No)
TS (once)
TS (twice)
VMO Specialty
Cardiothoracic Surg.
Colorectal Surgery
Endocrinology
Gastroenterology
General Medicine Phy
Gerontology
Gynecology
Hematology
Medical Oncology
Neurosurgery
Obstetrics \& Gynae
Orthopedic Surgery
Respiratory Medicine
Upper GI Surgery

OR:0.297,95\% CI (0.224,0.393), $\mathrm{P}<0.001$ OR:0.479,95\% CI $(0.353,0.65), \mathrm{P}<0.001$ OR:0.819,95\% CI $(0.673,0.997), \mathrm{P}=0.046$ OR:1.621,95\% CI (1.179,2.229), $\mathrm{P}=0.003$ OR:0.628,95\% CI $(0.511,0.771), \mathrm{P}<0.001$

OR:1.995,95\% CI (1.207,3.299), $\mathrm{P}=0.007$ OR:2.012,95\% CI (1.245,3.25), $\mathrm{P}=0.004$ OR:7.75,95\% CI $(3.52,17.063), \mathrm{P}<0.001$ OR:5.11,95\% CI $(1.844,14.155), \mathrm{P}=0.002$ OR:2.894,95\% CI (1.148,7.293), $\mathrm{P}=0.024$ OR:9.925,95\% CI (3.972,24.802), $\mathrm{P}<0.001$ OR:2.083,95\% CI (1.209,3.59), $\mathrm{P}=0.008$ OR:0.442,95\% CI $(0.247,0.791), \mathrm{P}=0.006$ OR:2.71,95\% CI (1.544,4.757), $\mathrm{P}=0.001$ OR:5.149,95\% CI $(2.545,10.414), \mathrm{P}<0.001$

OR:1.66,95\% CI (1.039,2.652), $\mathrm{P}=0.034$

OR:1.501,95\% CI (1.116,2.019), $\mathrm{P}=0.007$ OR:1.794,95\% CI $(1.264,2.547), \mathrm{P}=0.001$ OR:4.907,95\% CI (4.015,5.997), $\mathrm{P}<0.001$ OR:0.002,95\% CI $(0.002,0.003), \mathrm{P}<0.001$ OR:0.319,95\% CI $(0.276,0.369), \mathrm{P}<0.001$ OR:3.05,95\% CI $(2.209,4.211), \mathrm{P}<0.001$ OR:0.543,95\% CI (0.44,0.67), P<0.001

OR:2.341,95\% CI $(1.857,2.953), \mathrm{P}<0.001$ OR:0.726,95\% CI $(0.551,0.957), \mathrm{P}=0.023$

OR:0.182,95\% CI $(0.099,0.334), \mathrm{P}<0.001$ OR:0.272,95\% CI $(0.16,0.463), \mathrm{P}<0.001$ OR:0.392,95\% CI $(0.202,0.76), \mathrm{P}=0.006$ OR: $0.43,95 \%$ CI $(0.25,0.74), \mathrm{P}=0.002$ OR:0.32,95\% CI (0.191,0.538), $\mathrm{P}<0.001$ OR:0.265,95\% CI (0.146,0.479), $\mathrm{P}<0.001$ OR:0.13,95\% CI $(0.047,0.359), \mathrm{P}<0.001$ OR: $0.454,95 \%$ CI $(0.247,0.834), \mathrm{P}=0.011$ OR:0.383,95\% CI $(0.219,0.67), \mathrm{P}=0.001$ OR:0.262,95\% CI $(0.143,0.481), \mathrm{P}<0.001$ OR:0.271,95\% CI $(0.089,0.824), \mathrm{P}=0.021$ OR:0.399,95\% CI (0.232,0.687), $\mathrm{P}=0.001$ OR:0.405,95\% CI $(0.206,0.798), \mathrm{P}=0.009$ OR: $0.494,95 \%$ CI $(0.28,0.87), \mathrm{P}=0.015$ OR: $0.16,95 \%$ CI $(0.028,0.922), \mathrm{P}=0.04$ OR:0.524,95\% CI (0.294,0.935), $\mathrm{P}=0.029$
OR:0.15,95\% CI (0.079,0.283), $\mathrm{P}<0.001$
-
-

OR:0.508,95\% CI (0.267,0.965), $\mathrm{P}=0.039$

OR:2.481,95\% CI $(1.264,4.87), \mathrm{P}=0.008$ OR:7.051,95\% CI $(3.35,14.844), \mathrm{P}<0.001$

OR:2.632,95\% CI (1.231,5.629), $\mathrm{P}=0.013$

OR:3.952,95\% CI (1.41,11.081), $\mathrm{P}=0.009$ OR:0.031,95\% CI (0.006,0.164), $\mathrm{P}<0.001$ OR:0.358,95\% CI (0.181,0.708), $\mathrm{P}=0.003$

OR:5.521,95\% CI (4.289,7.108), $\mathrm{P}<0.001$ OR:0,95\% CI $(0,0.001), \mathrm{P}<0.001$

OR:0.039,95\% CI $(0.03,0.051), \mathrm{P}<0.001$

OR:2.464,95\% CI (1.232,4.93), $\mathrm{P}=0.011$ OR:4.688,95\% CI $(2.581,8.516), \mathrm{P}<0.001$

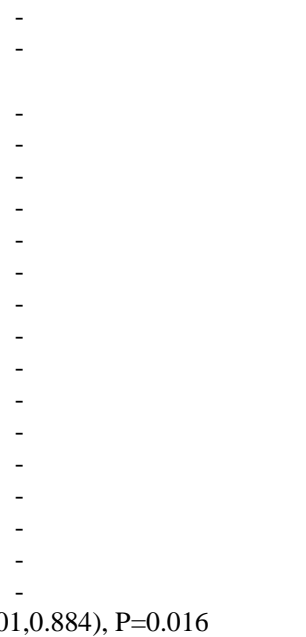

\subsection{Association between the Risk Factors}

The results of the Pearson correlation coefficient between the risk factors for the surgical and medical patients are shown in Table 5. Although the Pearson correlation coefficient for most of the features are negligible $(\mathrm{r}< \pm 0.3)$, there are a few others that show low $( \pm 0.3<r< \pm 0.5)$ and moderate $( \pm 0.5<r< \pm 0.7)$ correlations. Interestingly, the features considered in this study have a negligible correlation with the death rate of surgical and medical patients, which is in contrast with the findings of previous studies [11]. However, Age has a moderate correlation with Charlson Score, LOS has a low correlation with CHADx, HACs, ROR, and HTS for surgical patients, and negligible correlation with features considered for medical patients except CHADx with low correlation.

\subsection{Discussion}

This study identifies the predictors of ELOHS for surgical and medical patients, shows the correlation of these predictors amongst each other, and establishes how ELOHS influences CHADx for patients with different MDCs. The risk of ELOHS is very predominant for patients who are 40 years and over and have been treated with MDC such as "ear, nose, mouth \& throat", "male reproductive system", "circulatory system", "digestive system", "factors influencing health status" and 
"neoplastic disorders". Previously studies have also linked age to ELOHS [9] because of the increased vulnerability of the elderly to hospital-acquired infections and other complications in hospitals [18]. Unfortunately, with these complications and ELOHS, the hospitals can be hit with an increased cost of managing patients and shortage of available bed spaces for managing new patients [19-20]. ELOHS is also connected to the number of comorbidities, health complications, and socioeconomic status of patients [21-22]. Nonetheless, despite linking ELOHS to some of the MDCs identified earlier, socioeconomic status did not contribute to ELOHS. Admission Category (ADC) played a role in ELOHS due to the complications faced by patients under certain categories and the strategies used for managing them. It may therefore be important to adjust caregiving strategies for such ADC categories in consideration of other risk factors to improve patients' outcomes and reduce the LOS [23].
Even though patients that stayed $>20$ days in hospital posed a greater risk of ELOHS, there is the need to balance the management of those patients staying $<20$ days to forestall Hospital-acquired complications (HACs), which are the direct consequences of ELOHS [24-25]. Despite the importance of RRT calls in improving patients' status on hospital admission via moderating pulses, respiratory rates, blood pressures, oxygen saturation, etc. [26-27], it provided mixed results for surgical and medical patients. Thus, RRT calls did not influence ELOHS among surgical patients but those with no RRT calls have $90-369 \%$ more risk of ELOHS amongst medical patients than those with one or more RRT calls. So, the assumption that patients who have no RRT calls may be doing very well and may not overstay their expected LOS in the hospital may not always be accurate.

\begin{tabular}{|c|c|c|c|c|c|c|c|c|c|c|c|}
\hline a & क & 鱼 & $\underset{\pi}{\pi}$ & $\frac{\pi}{2}$ & $\stackrel{\mathcal{E}}{E}$ & $\stackrel{0}{0}$ & 索 & 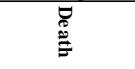 & 晃 & 5 & \\
\hline $0.555(<0.001)$ & $-0.063(<0.001)$ & $0.023(0.23)$ & $0.01(0.614)$ & $0.068(<0.001)$ & $0.029(0.131)$ & $-0.141(<0.001)$ & $-0.206(<0.001)$ & $0.031(0.106)$ & $0.014(0.475)$ & $0.037(0.052)$ & Age \\
\hline $0.231(<0.001)$ & $0.246(<0.001)$ & $0.261(<0.001)$ & $0.362(<0.001)$ & $0.475(<0.001)$ & $0.412(<0.001)$ & $-0.136(<0.001)$ & $0.019(0.314)$ & $0.168(<0.001)$ & $0.331(<0.001)$ & & LOS \\
\hline $0.06(0.002)$ & $0.031(0.102)$ & $0.1(<0.001)$ & $-0.074(<0.001)$ & $0.081(<0.001)$ & $0.081(<0.001)$ & $-0.404(<0.001)$ & $-0.2(<0.001)$ & $0.055(0.004)$ & & & HTS \\
\hline $0.185(<0.001)$ & $0.079(<0.001)$ & $0.2(<0.001)$ & $0.079(<0.001)$ & $0.153(<0.001)$ & $0.125(<0.001)$ & $-0.073(<0.001)$ & $-0.027(0.165)$ & & & & Death \\
\hline$-0.068(<0.001)$ & $-0.072(<0.001)$ & $-0.022(0.247)$ & $-0.039(0.044)$ & $0.118(<0.001)$ & $0.121(<0.001)$ & $0.3(<0.001)$ & & & \multirow{6}{*}{ 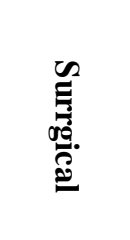 } & & AORT \\
\hline$-0.124(<0.001)$ & $-0.126(<0.001)$ & $-0.053(0.005)$ & $0.007(0.714)$ & $0(0.999)$ & $0.005(0.794)$ & & & & & & DOP \\
\hline $0.161(<0.001)$ & $0.087(<0.001)$ & $0.219(<0.001)$ & $0.214(<0.001)$ & $0.522(<0.001)$ & & & & & & & CHAD \\
\hline $0.22(<0.001)$ & $0.091(<0.001)$ & $0.276(<0.001)$ & $0.217(<0.001)$ & & & & & & & & HACs \\
\hline $0.093(<0.001)$ & $0.084(<0.001)$ & $0.096(<0.001)$ & & & & & & & & & ROR \\
\hline $0.088(<0.001)$ & $0.1(<0.001)$ & & & & & & & & & & RRT calls \\
\hline \multirow[t]{10}{*}{$0.074(<0.001)$} & & & & & & & & & & & TS \\
\hline & & & CS & RRT calls & TRAF & UPR & HACs & CHAD & Deaths & LOS & \\
\hline & & & $0.524(<0.001)$ & $0.02(<0.001)$ & $0.022(0.499)$ & $0.006(0.457)$ & $0.097(0.831)$ & $0.069(<0.001)$ & $0.043(0.018)$ & $0.095(0.143)$ & Age \\
\hline & & & $0.191(<0.001)$ & $0.165(<0.001)$ & $0.02(<0.001)$ & $-0.116(0.489)$ & $0.281(<0.001)$ & $0.301(<0.001)$ & $0.126(<0.001)$ & & LOS \\
\hline & & & $0.265(<0.001)$ & $0.051(<0.001)$ & $0.063(0.078)$ & $-0.076(0.03)$ & $0.159(0.009)$ & $0.141(<0.001)$ & & & Deaths \\
\hline & & & $0.157(<0.001)$ & $0.182(<0.001)$ & $0.078(<0.001)$ & $-0.038(0.007)$ & $0.494(0.188)$ & & & & CHAD \\
\hline & & & $0.176(<0.001)$ & $0.09(<0.001)$ & $0.078(0.002)$ & $-0.061(0.007)$ & & & \multirow{4}{*}{ 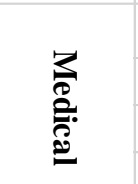 } & & HACs \\
\hline & & & $0.031(<0.001)$ & $0.025(0.296)$ & $0.133(0.388)$ & & & & & & UPR \\
\hline & & & $0.133(<0.001)$ & $0.061(<0.001)$ & & & & & & & TRAF \\
\hline & & & $0.045(<0.001)$ & & & & & & & & RRT calls \\
\hline
\end{tabular}

Psychosocial factors such as DTH and SES are not identified risk factors of ELOHS because they are not significant at a 95\% confidence interval, however, previous studies have linked the factors to ELOHS [23, 25 , 34]. Surgical patients treated for "female reproductive system" have between 28.06 - $497.98 \%$ more risk of ELOHS than patients treated for other MDCs. With the higher likelihood of ELOHS for "female reproductive system", "ear, nose, mouth and throat", "male reproductive system", and "eye disease and disorder" MDC patients than the other MDCs and risk factors, the need for strategic plans to manage these patients on admission and surgical procedures cannot be overemphasized. Similarly, Surgical patients that spent < 60 minutes in the operating theatre have a higher likelihood of ELOHS than those that stayed between 60 
- 120 minutes in the theatre. Although it may be premature to make conclusions about the reasons for this occurrence, research has previously linked 14-17\% hospital-acquired infections to surgical site infections resulting from endogenous and procedure-related risk factors [35]. It can also be inferred from the result that hospital factors such as VMO specialty e.g., hematology, vascular surgery, orthopedic surgery, medical oncology, endocrinology, neurology, colorectal surgery, neurosurgery, and general medical physician, have limited influence on ELOHS despite being risk factors. The risk of ELOHS amongst surgical patients also increased with age, a finding that is supported by previous studies [23, 25, 29].

Medical patients admitted with "ear, nose, mouth and throat" MDC have significantly higher ELOHS expectation than other patients with neoplastic disorder, digestive system, factors influencing health, respiratory system and musculoskeletal system and connected system MDCs. Charlson score and Intensive Care Unit (ICU) visit though are risk factors for ELOHS have limited likelihood of causing ELOHS.

\subsection{Conclusions}

This study looked at the hospital records of 21926 surgical and 11826 medical patients to identify the predictors of ELOHS using hospital and psychosocial factors. By using descriptive statistics, Chi-square, Pearson correlation, and multivariate Logistic regression, the risk factors of ELOHS were identified while establishing the prevalence of CHADx amongst ELOHS and NLOHS patients. It was found that $9.94 \%$ and $12.41 \%$ of medical and surgical patients respectively overstayed the high trim points of their DRGs. There were 22 MDCs for over 90 DGRs with the high trim points from 3 - 60 days considered in this study. The risk factors of ELOHS were identified as CS, AORT, CHADx, HACs, DOP, HTS, ICU transfer, LOS, TS, RRT call, ADC, and Age. Some MDCs such as "ear, nose, mouth \& throat", "male reproductive system", "circulatory system", "digestive system, "factors influencing health status", and "neoplastic disorders". were also identified as potential risk factors of ELOHS.

There is a difference in the prevalence of CHADx amongst ELOHS and NLOHS patients except for "kidney and urinary tract", "nervous system", "circulatory system" and "ear, nose, mouth \& throat" MDCs. There is a moderate correlation between patients' age and the Charlson Score and CHADx and HACs while many other relationships amongst the features are low and negligible. It will be important to consider the risk factors in managing patients of different MDCs and DRG vulnerabilities to improve their health outcomes within the expected LOS. This will go a long way to reduce the cost of managing patients and improve their quality-oflife.
The limitations of this study include the reporting accuracy of inputted and extracted data from the database and the data size of some MDCs. There may be the need to study the MDCs separately to have a better picture of the predictors' behaviour. Finally, despite the DRGs being identified separately, it may be important to have better granular information about them to facilitate analysis that will identify predictors of ELOHS for different diagnosis severity.

\section{Acknowledgment}

The authors wish to thank the Digital Health Cooperative Research Centre (DHCRC) for the financial supports and other contributions that made this research possible.

\section{References}

[1]. S. Benenson, M.J. Cohen, C. Schwartz, M. Revva, A.E Moses, and P.D. Levin, "Is it financially beneficial for hospitals to prevent nosocomial infections?". BMC Health Services Research, 20(1), 2020, pp.1-9.

[2]. Y. Cai, M. Zhu, W. Sun, X. Cao, and H. Wu, "Study on the cost attributable to central venous catheter-related bloodstream infection and its influencing factors in a tertiary hospital in China", Health and quality of life outcomes, 2018, 16(1), pp.1-6.

[3]. A.B. Koo, A.A. Elsamadicy, I.H. Lin, W.B. David, N. Sujijantarat, C. Santarosa, B.J. Cord, A. Zetchi, R. Hebert, F. Bahrassa, and A. Malhotra, "Predictors of Extended Length of Stay Following Treatment of Stay Following Treatment of Unruptured Adult Cerebral Aneurysms: A Study of The National Inpatient Sample. Journal of Stroke and Cerebrovascular Diseases, 29(11), p.105230.

[4]. P.W. Stone, "Economic burden of healthcare-associated infections: an American perspective", Expert review of pharmacoeconomics \& outcomes research, 9(5), 2009, pp.417422.

[5]. H.A. Khan, F.K. Baig, and R. Mehboob, "Nosocomial infections: Epidemiology, prevention, control and surveillance", Asian Pacific Journal of Tropical Biomedicine, 7(5), 2017, pp.478-482.

[6]. L. Deeter, M. Seaton, G.J. Carrougher, K. McMullen, S.P. Mandell, D. Amtmann, and N.S. Gibran, "Hospital-acquired complications alter quality of life in adult burn survivors: report from a burn model system”, Burns, 45(1), 2019, pp.42-47.

[7]. S. Boes, and C. Napierala, "Assessment of the introduction of DRG-based reimbursement in Switzerland: Evidence on the short-term effects on length of stay compliance in university hospitals", Health Policy, 2021.

[8]. T. Jaqua, and E. Jaqua, "Factors Affecting Hospital Reimbursements", J Nurs Healthcare Manage, 2, 2019, p.103.

[9]. P.G. Passias, C.M. Jalai, N. Worley, S. Vira, S. Hasan, S.R. Horn, F.A. Segreto, C.A., Bortz, A.P. White, M. Gerling, and V. LaFage, "Predictors of hospital length of stay and 30-day readmission in cervical spondylotic myelopathy patients: an analysis of 3057 patients using the ACS-NSQIP database", World neurosurgery, 110, 2018, pp.e450-e458.

[10]. J. Deister, B.G. Cothern, C. Williams, A.W. Froehle, and R.T. Laughlin, "Factors predicting length of hospital stay and extended care facility admission after hindfoot arthrodesis procedures", The Journal of Foot and Ankle Surgery, 56(4), 2017, pp.805-812. 
[11]. K.J. Ho, A.L. Madenci, J.T. McPhee, M.E. Semel, R.A. Bafford, L. L. Nguyen, C.K. Ozaki, and M., Belkin, "Contemporary predictors of extended postoperative hospital length of stay after carotid endarterectomy". Journal of vascular surgery, 59(5), 2014, pp.1282-1290.

[12]. A. Storey, B. MacDonald, and M.A. Rahman, "The association between preoperative length of hospital stay and deep sternal wound infection: A scoping review", Australian Critical Care, 2021.

[13]. S. Tal, "Length of hospital stay among oldest-old patients in acute geriatric ward" Archives of Gerontology and Geriatrics, 94, 2021, p.104352.

[14]. M.J. McGirt, S.L. Parker, S. Chotai, D. Pfortmiller, J.M. Sorenson, K. Foley, and A.L. Asher, "Predictors of extended length of stay, discharge to inpatient rehab, and hospital readmission following elective lumbar spine surgery: introduction of the Carolina-Semmes Grading Scale", Journal of Neurosurgery: Spine, 27(4), 2017, pp.382-390.

[15]. E.O. Klineberg, P.G. Passias, C.M. Jalai, N. Worley, D.M. Sciubba, , D.C. Burton, M.C. Gupta, A. Soroceanu, A., P.L. Zebala, G.M. Mundis Jr, and H.J. Kim, "Predicting extended length of hospital stay in an adult spinal deformity surgical population", Spine, 41(13), 2016, pp.E798-E805.

[16]. T.L. Flanigan, E.M. Kiskaddon, J.A. Rogozinski, M.D. Thomas, A.W. Froehle, and A.B. Krishnamurthy, "Predictive Factors of Extended Length of Hospital Stay Following Total Joint Arthroplasty in a Veterans Affairs Hospital Population”, The Journal of Arthroplasty, 36(5), 2021, pp.1527-1532.

[17]. ABS (Australian Bureau of Statistics), An introduction to socio-economic indexes for areas (SEIFA), Commonwealth of Australia, ABS, Canberra, 2011. Available from https://www.abs.gov.au/websitedbs/censushome.nsf/home/s eifa 26/05/2021

[18]. M. Avci, O. Ozgenc, S.A. Coskuner, and A.I. Olut, "Hospital acquired infections (HAI) in the elderly: comparison with the younger patients", Archives of gerontology and geriatrics, 54(1), 2012, pp.247-250.

[19]. H.J. Toh, Z.Y. Lim, P. Yap, and T. Tang, "Factors associated with prolonged length of stay in older patients", Singapore medical journal, 58(3), 2017, p.134.

[20]. P. Hendy, J.H. Patel, T. Kordbacheh, N. Laskar, and M. Harbord, "In-depth analysis of delays to patient discharge: a metropolitan teaching hospital experience", Clinical Medicine, 12(4), 2012, p.320.

[21]. M. Bo, G. Fonte, F. Pivaro, M. Bonetto, C. Comi, V. Giorgis, L. Marchese, G. Isaia, G. Maggiani, E. Furno, and Y. Falcone, "Prevalence of and factors associated with prolonged length of stay in older hospitalized medical patients", Geriatrics \& gerontology international, 16(3), 2016, pp.314-321.

[22]. B.A. Marfil-Garza, P.F. Belaunzarán-Zamudio, A. GuliasHerrero, A.C. Zuñiga, Y. Caro-Vega, D. KershenobichStalnikowitz, and J. Sifuentes-Osornio, "Risk factors associated with prolonged hospital length-of-stay: 18-year retrospective study of hospitalizations in a tertiary healthcare center in Mexico", PloS one, 13(11), 2018, p.e0207203.

[23]. M.E. Anderson, J.J. Glasheen, D. Anoff, R. Pierce, R. Capp, and C.D. Jones, "Understanding predictors of prolonged hospitalizations among general medicine patients: A guide and preliminary analysis", Journal of hospital medicine, 10(9), 2015, pp.623-626.

[24]. S.P. Kim, N.D. Shah, R.J. Karnes, C.J. Weight, I. Frank, J.P. Moriarty, L.C. Han, B. Borah, M.K. Tollefson, and S.A. Boorjian, "The implications of hospital acquired adverse events on mortality, length of stay and costs for patients undergoing radical cystectomy for bladder cancer", The Journal of urology, 187(6), 2012, pp.2011-2017.

[25]. N. Kumar, R.S. Patel, S.S.Y. Wang, J.Y.H. Tan, A. Singla, Z. Chen, N. Ravikumar, A. Tan, N. Kumar, D.H.W. Hey, and S.V. Prasad, "Factors influencing extended hospital stay in patients undergoing metastatic spine tumour surgery and its impact on survival", Journal of Clinical Neuroscience, 56, 2018, pp.114-120.

[26]. C.L. Downey, W. Tahir, R. Randell, J.M. Brown, and D.G. Jayne, "Strengths and limitations of early warning scores: a systematic review and narrative synthesis", International Journal of Nursing Studies, 76, 2017, pp.106-119.

[27]. S. Crouch, L.G. Trahair, and L.M. Aitken, "The use of altered rapid response calling criteria in a tertiary referral facility", Australian Critical Care, 34(3), 2021, pp.204-208.

[28]. R.W. Krell, M.E. Girotti, and J.B. Dimick, "Extended length of stay after surgery: complications, inefficient practice, or sick patients?", JAMA surgery, 149(8), 2014, pp.815-820.

[29]. Australian Institute of Health and Welfare, Health expenditure Australia 2018-19. Health and Welfare expenditure series no.66. Cat. no. HWE 80. Canberra: AIHW, 2020, Available from < https://www.aihw.gov.au/getmedia/a5cfb53c-a22f-407b8c6f-3820544cb900/aihw-hwe-80.pdf.aspx?inline=true > on 9/06/2021

[30]. B.L. Dial, V.R. Esposito,.R. Danilkowicz, J. O’Donnell, B. Sugarman, D.J. Blizzard and M. E. Erickson, "Factors associated with extended length of stay and 90-day readmission rates following ACDF". Global spine journal, 10(3), 2020, pp.252-260.

[31]. A. A. Elsamadicy, A.B. Koo, A.J. Kundishora, F. Chouairi, M. Lee, A.C. Hengartner, J. Camara-Quintana, K. T. Kahle, and M. L. DiLuna. "Impact of patient and hospital-level risk factors on extended length of stay following spinal fusion for adolescent idiopathic scoliosis". Journal of Neurosurgery: Pediatrics, 24(4), 2019, pp.469-475.

[32]. K.M. Trentino, S. G. Swain, S.A. Burrows, P. C. Sprivulis and F. F. Daly, "Measuring the incidence of hospitalacquired complications and their effect on length of stay using CHADx". Medical Journal of Australia, 199(8), 2013, pp.543-547.

[33]. Australian Commission on Safety and Quality in Healthcare (ACSQH), Classification of Hospital Acquired Diagnoses, available from < https://www.safetyandquality.gov.au/ourwork/indicators/classification-of-hospital-acquireddiagnoses $>23 / 08 / 2021$

[34]. A. K. Ghosh, B. P. Geisler and S. Ibrahim, S., "Racial/ethnic and socioeconomic variations in hospital length of stay: A state-based analysis. Medicine, 100(20), 2021.

[35]. A. M. Spagnolo, G. Ottria, D. Amicizia, F. Perdelli and M. L. Cristina, "Operating theatre quality and prevention of surgical site infections". Journal of preventive medicine and hygiene, 54(3), 2013, p.131. 